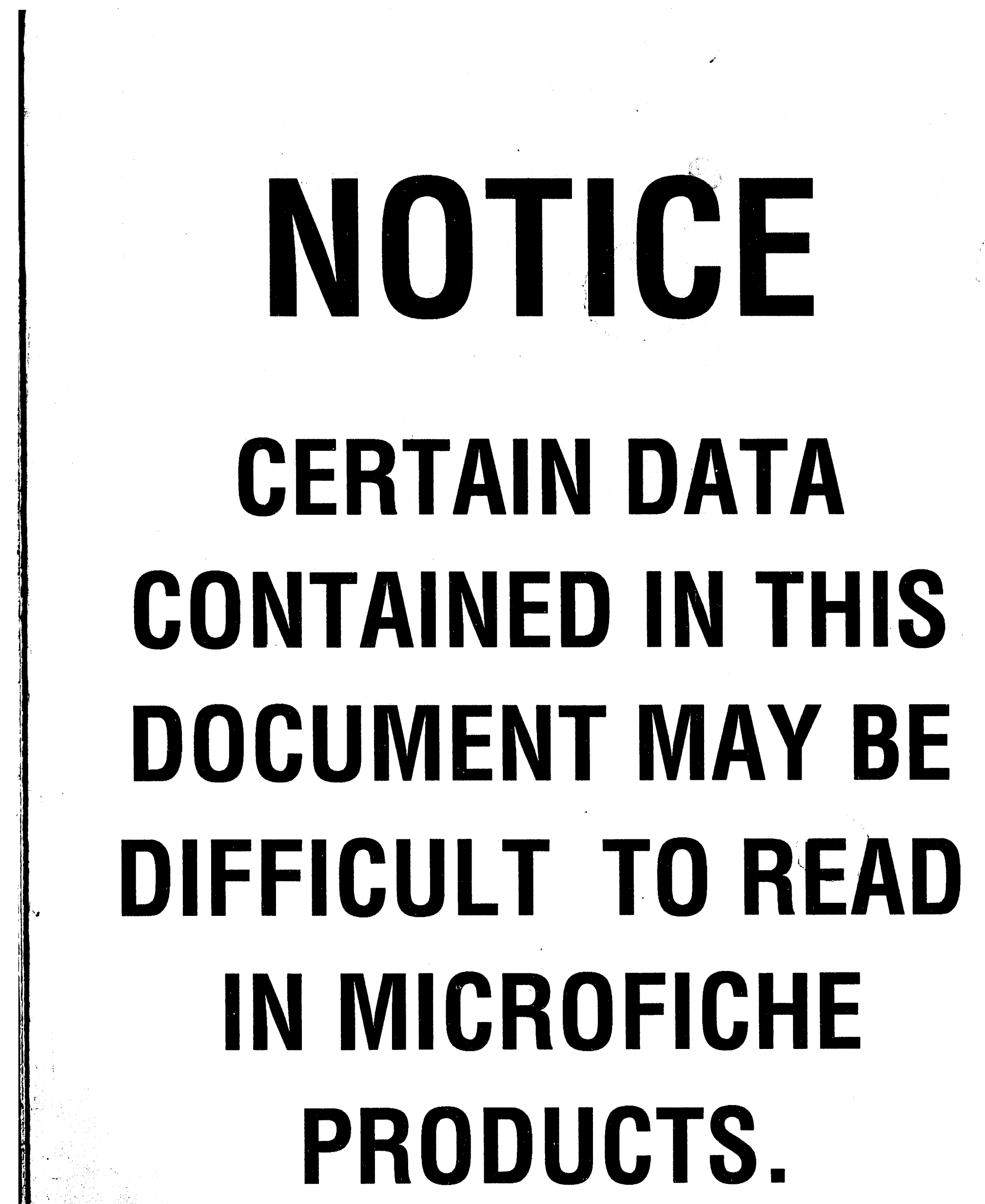




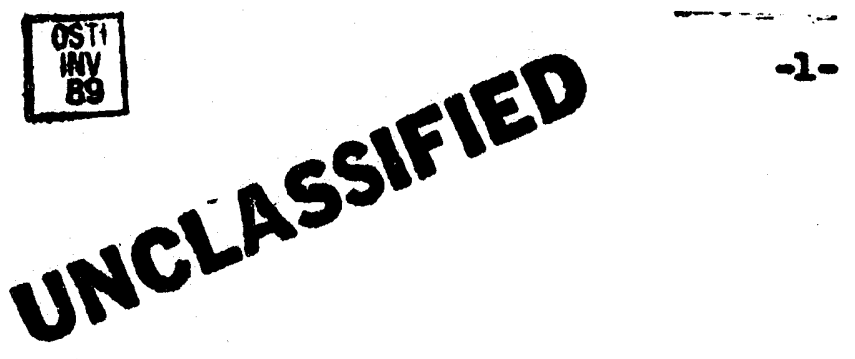

Atonito Thores Counteaten

Fanferd Oparation Cerlos

Rtahiand, Hashington

Attentiens Mr. D.2. Bhaw, Mnager

Gentilem:

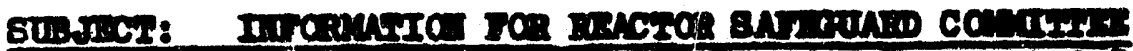

Ber: Itocanim, B.W. Cook to D.J. Bhw, dated 2/15/52, cis $-19,565$

Subuttted In Itended fors arrenged to bateh the greations of tho

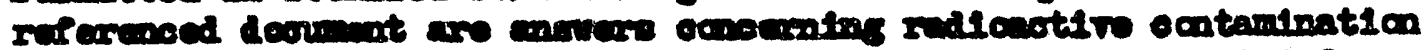
in the deinity of the Fanford Worb. Incen anta are intended for ro-

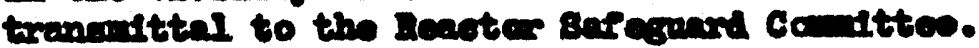

If an addt1enal inta are roquired, No. I.J. Pas, who propared the

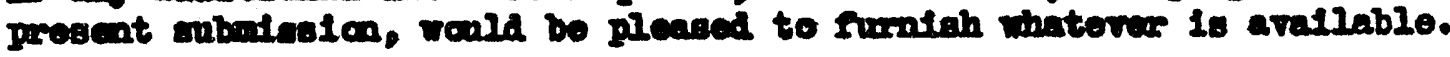

Texy truls jours,

ORIGINAL SIGNED

BY H. M. PARKER

IM Parkersento

Directar

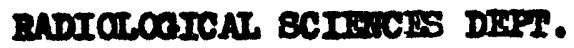

13341

Pition

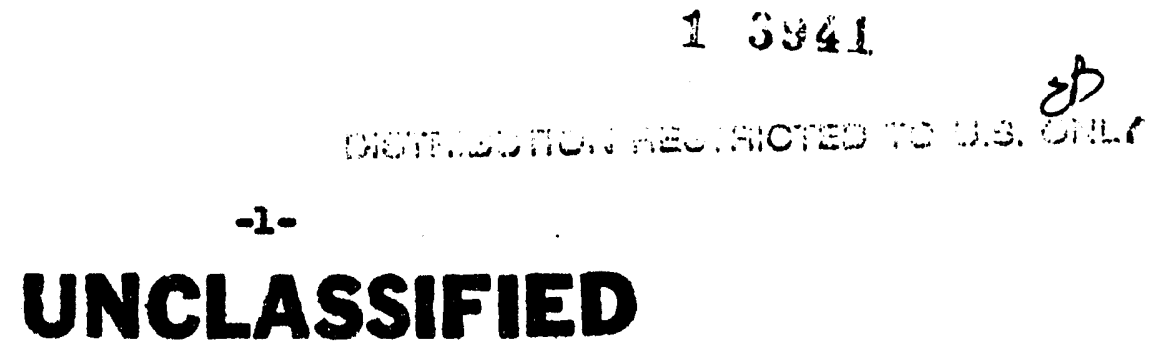


Iten 1

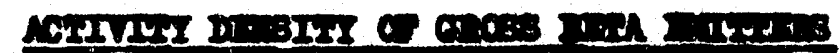

II.

Menth

1951

Jumary

Jobruavy

Marah

Apre11

Int

June

Juls

Ansuet

Soptember

Ootaber

Ioverber

Deocubar

1952

Jameary

Iobruary

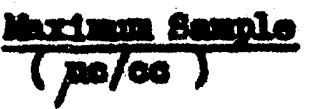

Donthl Arease (molec)
$4.1 \times 10^{-6}$
$7.4=10^{-6}$
$7.7=10^{-6}$
$8.7=10^{-6}$
$4.3=10^{-6}$
$2.5 \times 10^{-6}$
$2.1 \times 10^{-6}$
$7.3 \times 10^{-6}$
$1.1 \times 10-8$
$8.0=10^{-6}$
$8.5 \times 10^{-6}$
$9.6 \times 10^{-6}$
$7.9 \times 10^{-6}$
$1.0 \times 10^{-5}$

$1.3 \times 10^{-6}$

$3.8 \times 10^{-6}$

$6.0 \times 10^{-6}$

5.5. $\times 10^{-6}$

$5.6 \times 10^{-6}$

$5.4 \times 10^{-6}$

$5.4=10^{-6}$

$5.9 \times 10^{-6}$

\section{Item 2}

The maximum sample and maximin monthly aryrage activity denaity of groas beta

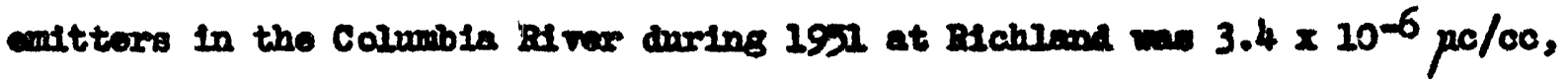
and $2.8 \times 10^{-6} \mathrm{po} / \mathrm{oc}$, reopectively. simdine meanremente obtained at the Pasco

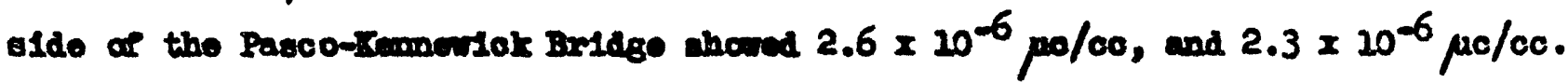

Item 3

Iow rates of the Columbia Alver as mannred on the Plrat day of the month from July, 1951, to date wore as follores

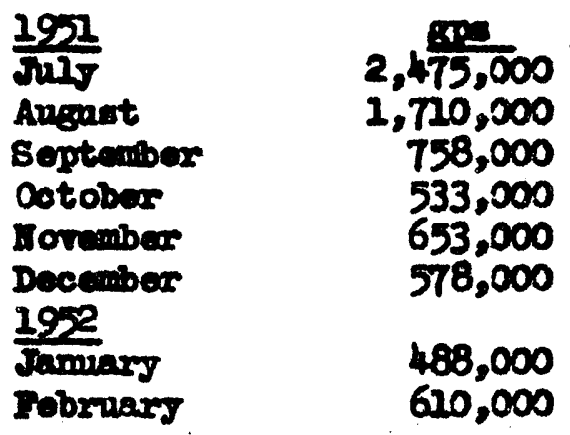



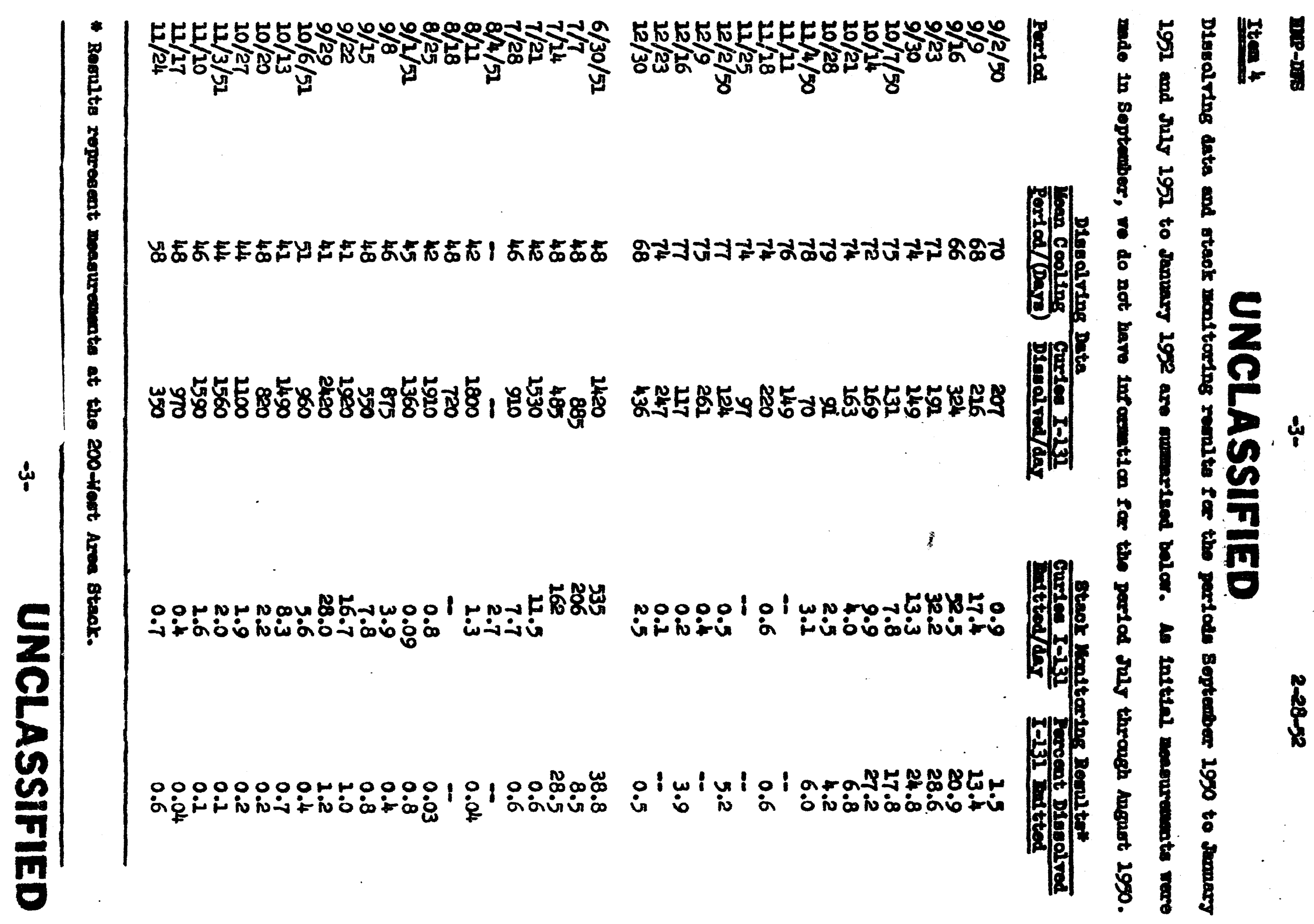
Ite. 4-Continued

UNCLASSIFIED

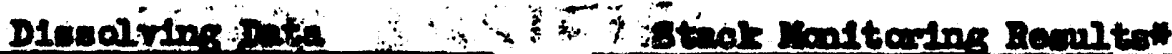

Peariod

12/1/51

$12 / 8$

12/15

12/22

$12 / 29$

$1 / 5 / 52$
Conces I-I3I

pregar redor cwe10 I-131

mtedidar

Pereent Dinalved

I-131 Eitted

$\begin{array}{rr}45 & 1630 \\ 43 & 1890 \\ 43 & 1150 \\ 47 & 905 \\ 54 & 55 \\ 45 & 1330\end{array}$

0.1

0.07

0.5

0.3

0.3

0.08

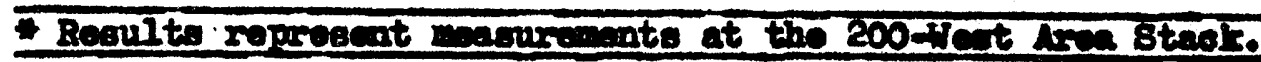

Item 5

The average activity density from $I^{131}$ a vegetintion calleoted from the locations requested frem Jannary, 1951, to date is tabulated belows

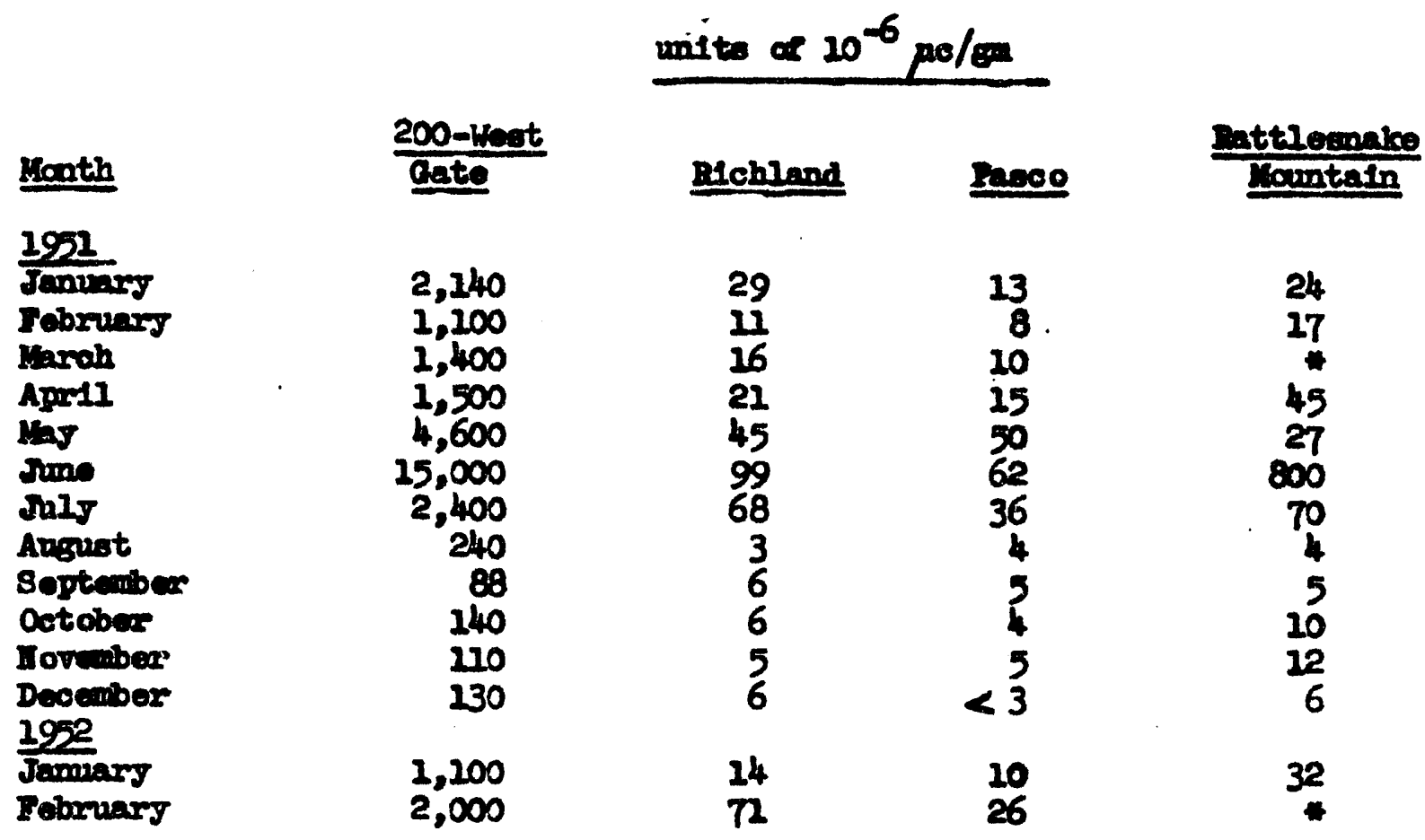

Location vas not sampled during this pariod. 


\section{Item 6}

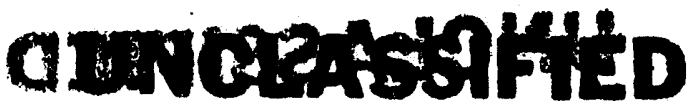

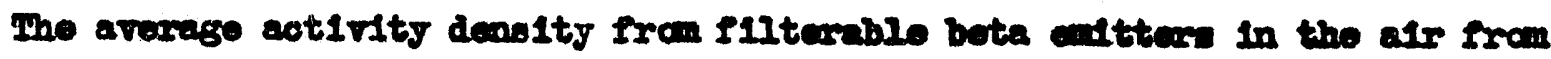
July, 1951, to date, at the locations requented, In marised belor:

$$
\text { unite of } 10^{-2 / 4} \text { pe/co }
$$

\begin{tabular}{|c|c|c|c|c|}
\hline Konth & $\frac{200 \text {-Weet }}{\text { Cate }}$ & Piohland & Paroo & $\frac{\text { cablo }}{\text { rountain }}$ \\
\hline 1951 & & & & \\
\hline $\begin{array}{l}\text { July } \\
\text { Ausust } \\
\text { Soptember } \\
\text { Ootober * } \\
\text { Iovember * } \\
\text { Decomber * } \\
1952\end{array}$ & $\begin{array}{r}579 \\
31 \\
51 \\
126 \\
128 \\
60\end{array}$ & $\begin{array}{r}39 \\
12 \\
167 \\
56 \\
91 \\
13\end{array}$ & $\begin{array}{l}49 \\
18 \\
19 \\
89 \\
58 \\
25\end{array}$ & $\begin{array}{r}38 \\
20 \\
24 \\
86 \\
116 \\
45\end{array}$ \\
\hline $\begin{array}{l}\text { Jomary } \\
\text { Fobruary }\end{array}$ & $\begin{array}{l}150 \\
199\end{array}$ & $\begin{array}{l}45 \\
22\end{array}$ & $\begin{array}{l}18 \\
10\end{array}$ & $\begin{array}{l}13 \\
28\end{array}$ \\
\hline
\end{tabular}

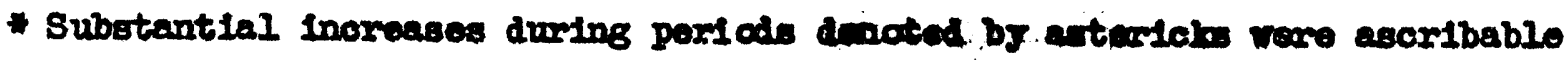

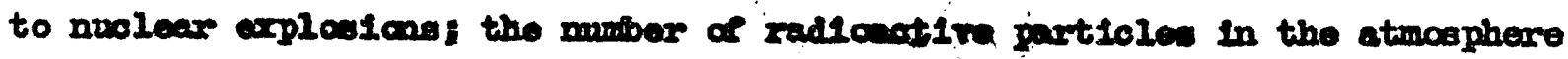
In the Paolfle Jarthrest renged from 1 to 10 partioles per ouble woter in 10olated cases during these throe months. (Doc.tur-23517 - "N Bopont of Particle Contanination, October-Decenber 1951", dated 2/15/52). 

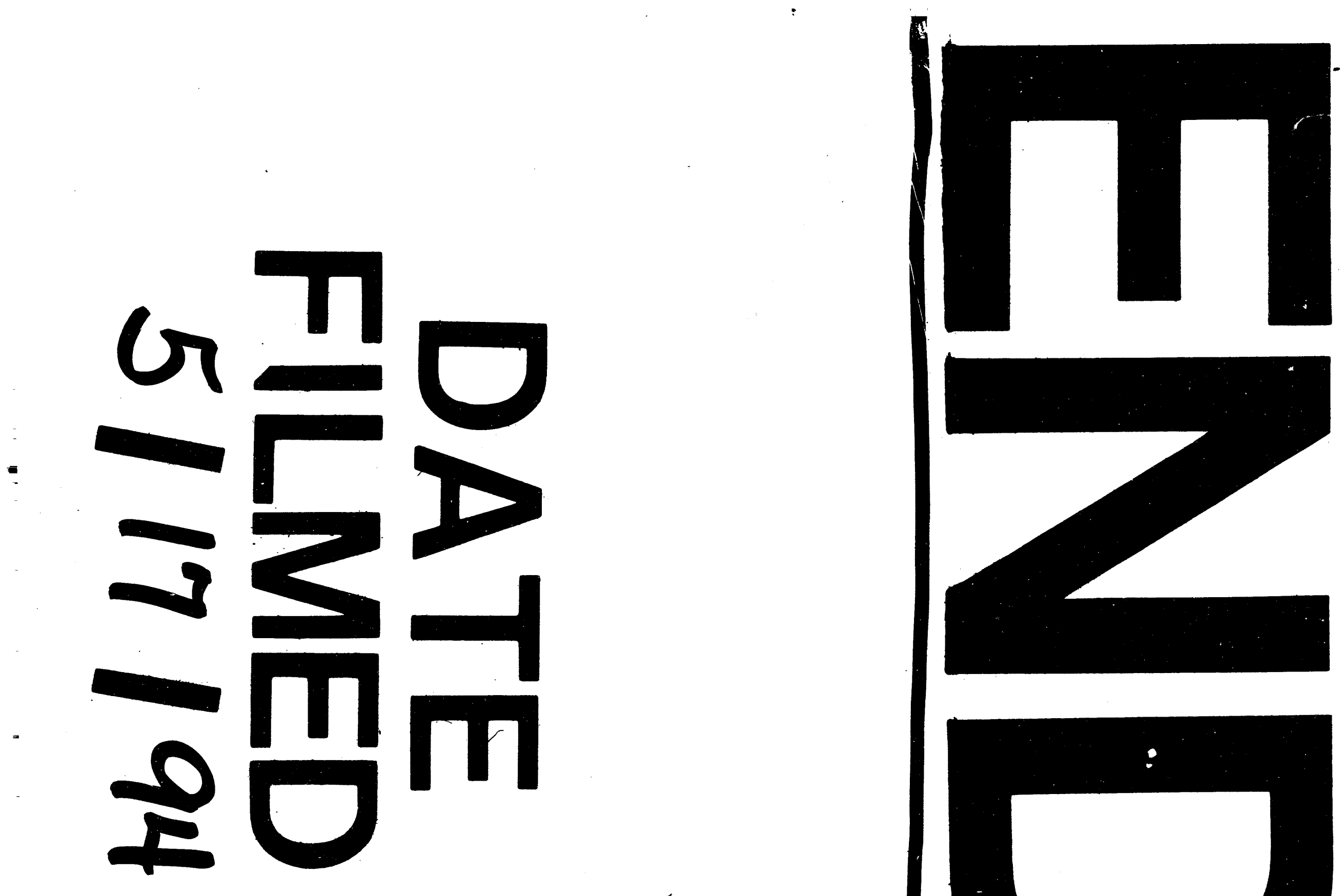

1

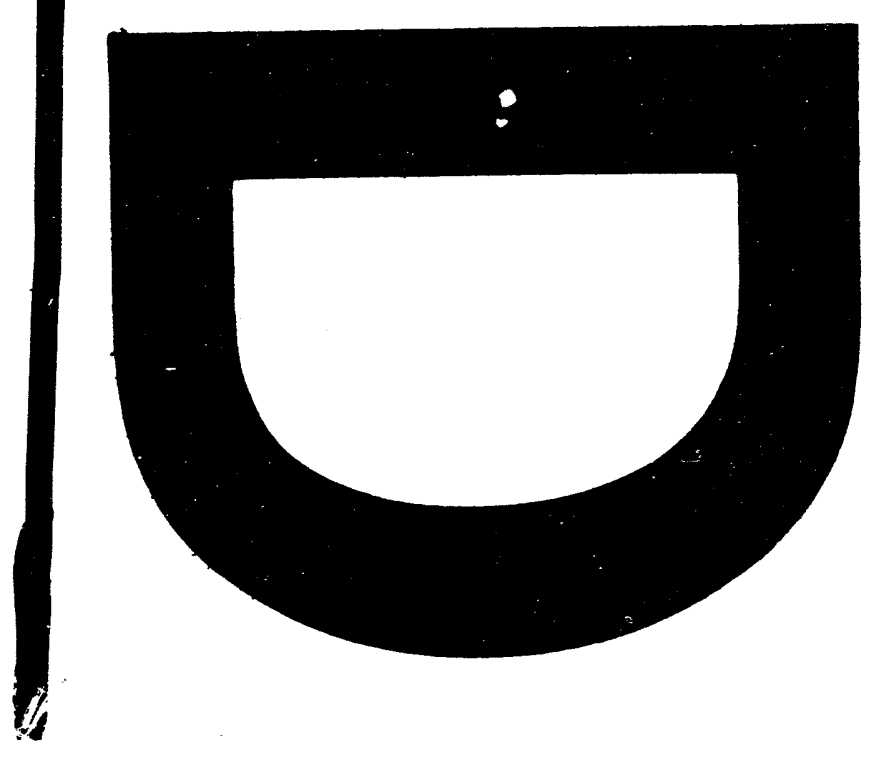

1 
